Report: LLNL-TR-577352

\title{
TART2012 \\ An Overview of \\ A Coupled Neutron-Photon 3-D, Combinatorial Geometry Time Dependent Monte Carlo Transport Code
}

\author{
by \\ Dermott E. Cullen \\ Lawrence Livermore National Laboratory \\ P.O. Box 808 \\ L-198 \\ Livermore, CA 94550
}

U.S. Department of Energy

July 22, 2012

Livermore

National

Laboratory

Approved for public release; further dissemination unlimited. 


\section{DISCLAIMER}

This document was prepared as an account of work sponsored by an agency of the United States government. Neither the United States government nor Lawrence Livermore National Security, LLC, nor any of their employees makes any warranty, expressed or implied, or assumes any legal liability or responsibility for the accuracy, completeness, or usefulness of any information, apparatus, product, or process disclosed, or represents that its use would not infringe privately owned rights. Reference herein to any specific commercial product, process, or service by trade name, trademark, manufacturer, or otherwise does not necessarily constitute or imply its endorsement, recommendation, or favoring by the United States government or Lawrence Livermore National Security, LLC. The views and opinions of authors expressed herein do not necessarily state or reflect those of the United States government or Lawrence Livermore National Security, LLC, and shall not be used for advertising or product endorsement purposes.

This work performed under the auspices of the U.S. Department of Energy by Lawrence Livermore National Laboratory under Contract DE-AC52-07NA27344. 


\title{
TART2012 \\ An Overview of \\ A Coupled Neutron-Photon \\ 3-D, Combinatorial Geometry \\ Time Dependent \\ Monte Carlo Transport Code
}

\author{
by \\ Dermott E. Cullen \\ Lawrence Livermore National Laboratory \\ P.O. Box 808 \\ L-198 \\ Livermore, CA 94550
}

July 22, 2012

\section{Contact}

I, Dermott E. Cullen, have been retired from Lawrence Livermore National Laboratory for a number of years. Presently I work part time for AKIMA, a private company that provides contract workers to LLNL. All of my work through AKIMA is performed at my private residence and I am rarely located on-site at LLNL. I rarely check my old Lab phone number or e.mail for messages. Therefore the most reliable way to contact me is at my home [12]. 


\begin{abstract}
This document is designed to provide an overview of TART2012, a coupled neutronphoton, 3 Dimensional, combinatorial geometry, time dependent Monte Carlo radiation transport code. This code can run on any modern computer. It is a complete system to assist you with input preparation, running Monte Carlo calculations, and analysis of output results. TART2012 is also incredibly FAST; if you have used similar codes, you will be amazed at how fast this code is compared to other similar codes. Use of the entire system can save you a great deal of time and energy.
\end{abstract}

This document is NOT A USER'S MANUAL for TART; indeed there is nothing in this document that describes how to use TART. This document merely briefly provides an overview of the history and capability of TART. The actual code user's manual remains the documentation for the earlier published code TART2005.

TART2012 is distributed on DVD. This DVD contains on-line documentation for all codes included in the system, the codes configured to run on a variety of computers, and many example problems that you can use to familiarize yourself with the system.

TART2012 completely supersedes all older versions of TART, and it is strongly recommended that users only use the most recent version of TART2012 and its data files.

\title{
Overview
}

The last version of TART published was TART2005 [r1], some seven years ago, while I was employed at Lawrence Livermore National Laboratory (LLNL). Since that time I retired from LLNL and there has been no code development or maintenance on this code at LLNL. All of the improvements since that time were performed by TART users throughout the World. These users have kindly freely supplied me with their improvements and I privately incorporated them into the code that today I call TART2012 and briefly describe here. I say briefly describe because much of the TART2005 documentation is still relevant and I plan to distribute it with TART2012.

Although I have retired from LLNL, I am presently a part time employee of AKIMA, a private company that provides contract workers to LLNL. In this capacity I have worked for Criticality Safety on a number of projects, none of them involving TART. Although none of the work on TART2012 was performed at LLNL, David Heinrichs has kindly agreed to support publication of this report through LLNL, in order to reach the widest possible audience. 


\section{Acknowledgments}

First I must acknowledge the major contributions of Fabrice Pelestor (DGA/Technique Naval, Toulon, France); he contributed many improvements to TART, particularly he contributed the 64 bit and multi-processing version, to greatly modernize the code and allow it to be efficiently used with today's computers. The code we have today, TART2012, could not have been produced without his contributions.

I thank Dave Heinrichs (LLNL) for supporting publication of this report, and agreeing to distribute TART2012 within LLNL. Next I acknowledge the cooperation with other Monte Carlo code designer/users, in particular, Bob MacFarlane (LANL, MCNP), Rich Procassini (LLNL, MERCURY), Ed Lent (LLNL,COG) and Maurice Greene (ORNL); coordinated code comparisons have led to improvements in TART as well as ALL of these other codes. I cannot stress enough that modern transport codes are far too complex to allow true code verification without detailed comparisons to other similar codes.

I also thank the many users of earlier versions of TART who have supplied extremely useful feedback to me. Since the general release of TART2005, the response from users in terms of feedback has been extremely useful in improving the code. These improvements have been in terms of correcting problems in the earlier releases of TART, and in terms of users proposing new or improved options to meet their needs, now incorporated in TART2012. Today virtually ALL improves to TART are based on feedback to me from TART users. I highly encourage all users to supply their feedback to me.

\section{New in TART2012 System}

Here is a quick summary of what's now included in TART2012 compared to the last published version TART2005; the summary is by year (this summary is also included inside the TART2012 code as comments)

2006

1) Corrected $S(a, b)$ coherent scatter to, scatter in the Lab (not CM) system. coherent scatter with no change speed.

2) Updated neutrons/fission sampling to include correction to conserve $\langle$ nu $>$.

3) Corrected criticality flux by material normalize

Now EXACTLY = Analog fission neutrons produce so they sum to total analog flux.

4) Missive change to correct reactall and reacted.

Now only analog - output also by ZA/C.

5) Added $\mathrm{C}=47-54$ charged particle reactions 
(currently only $\mathrm{C}=48$ in $\mathrm{ZA}=30000$ ).

6) Changed weights to relative - insure each source particle (neutron/photon) has a total weight $=1$

7) Removed bias and biasg - only way to insure the total weight $=1$

2007

1) Corrected high energy exponential spectra fit

2) Added stack to build for photon re-entry problem

3) High energy fission fit now $E^{*} \operatorname{Exp}\left[-a^{*} E\right]$

4) Low energy fission fit sqrt(E) to higher energy

2008

1) Spectra sqrt(E) below $10 \mathrm{keV}$.

2) $\left(n, n^{\prime}\right)$ neat threshold, low energy extension.

3) Updated NEWCROSS for ENDF/B-VII U238.

4) Updated for over 1,000,000 zones; extended output format.

5) Corrected cneutal and cphotal (was ignored)

6) Sentl 60 Intermediate Resonances now standard

\section{2}

1) Complete rewrite to incorporate all user feedback.

2) Made 64 bit compiler compatible.

3) Eliminated re-entrant coding.

4) Neutrons and photons sources now treated same.

5) sentl $1=3$ (neutron AND photon sources) is no longer allowed

6) Because of the above, sentl 18 photon/neutron source ratio no longer has any meaning.

7) Data files updated based on ENDF/B-VII.1 data.

It is important to note that through all of these updates, the TART input format has NOT BEEN CHANGED in any significant way, so that the INPUT documentation for TART2005 is still relevant and is distributed with TART2012.

The remainder of this document is more or less a copy of the TART2005 document (r1), with changes only where it was necessary to reflect changes in TART2012 or my status as retired. 


\section{2 versus 64 Bit Executables}

TART2012 is $100 \% 32$ versus 64 bit compatible; not one line of coding need be changed to create either 32 or 64 bit executables. But so far I have not found any significant advantage of 64 bit executables, except for very large problems that exceed the 4 GB, 32 bit limit. In deciding what is BEST FOR YOUR USE here are a few points to consider,

1) For the same problem both 32 and 64 bit executables give EXACTLY the same answer; so in terms of accuracy there is no advantage to one versus the other.

2) A 32 executable is slightly faster; this surprised me; a definite advantage to 32 bit executables.

3) 32 bit executables run on either 32 or 64 bit computers. In contrast 64 bit executables only run on 64 bit computer; another advantage for 32 bit executables.

The bottom line is that at least so far I have not found any significant advantage to using 64 bit executables rather than 32 bit. If YOU, THE CODE USER finds an advantage PLEASE tell me.

\section{New in TART2005 System}

Here is a quick summary of what's new in TART2005,

1) Continuous energy cross sections, including multi-band self-shielding in the unresolved resonance energy region.

2) Delayed neutron, time and energy distributions.

3) Improved thermal scattering law, $S(a, b)$, data.

4) Improved fission spectra.

5) Improved sub-critical system simulation.

6) Alpha static criticality calculations.

7) Output summary of events by reaction type (capture, $n, 2 n$, etc.), as well as neutron production and removal.

8) Output complete comparison of expected and analog results.

9) General corrections and improvements, particularly with regard to checking input parameters.

In addition, TART now uses the "BEST" physics concept, to set ALL input default values to provide users with the "BEST" available physics. With this concept users need only prepare the minimum TART input, focusing on the physics problems they are interested in, and relying on TART to provide the "BEST" available physics to meet their needs.

WARNING - users should review their older TART input decks to insure that they do not include options that override the default "BEST" values; in which case if you wish to use the "BEST" options, they need merely delete the now extraneous input parameters from their TART input decks. 


\section{The TART2005 System}

This report is intended merely as a brief introduction to TART2005. The on-line documentation for the TART2005 system codes, distributed on TART2005 DVD, has been coordinated to illustrate combined use of the codes to make your job simpler and your work easier to accomplish, in particular extensive use of interactive graphics. If you have not used interactive graphics before you are only making your job harder and your tasks will take longer to accomplish.

\section{Overview of This Report}

2012 Update: ALL of the documentation described here is still relevant and is planned to be distributed with the TART2012 DVD. The remainder of this section is copied from the TART2005 report.

This report describes all major changes in TART since TART95. As such this report supersedes the reports of TART96 [2], TART97, TART98, TART2000, and TART2002 [8a,b,c,d]. However, the large TART95 report [1] is still the most comprehensive report on TART.

This report is divided into a number of parts, with each part describing one part of the TART2005 DVD system. The parts are,

Part 1: TART2005 - Monte Carlo Calculations

Part 2: Input Parameters - Description of all Input Parameters

Part 3: TARTCHEK - Check TART Input and Display TART Results

Part 4: TARTAID - Create TART Input

Part 5: EPICSHOW - Display Atomic and Nuclear data used by TART

Part 6: PLOTTAB - General Plotting Code to Display TART Output

Part 7: EDITOR - Text editor for use with TART

Part 8: Utility Codes - A collection of Useful Codes

Part 9: IMAGES - Using TART for medical and industrial imaging

Part 10: Criticality - Using TART to calculate critical systems

I Strongly Recommend that you read the on-line documentation for all parts of this system, to get a better overall picture of how this entire code system fits together and can help you. The TART2005 on-line documentation is available in Microsoft Word and Adobe PDF formats, and includes black and white as well as color graphic results. Only when you start using the codes in combination will you realize that this is a complete system that can really assist you in your work.

\section{Computer Requirements}

TART2005 will run on any modern Computer, with at Least 20 Megabytes Memory and 60 Megabytes Disk Space. Dynamic memory management allows TART to run problems on virtually any size computer; up to 4 GB for 32 bit executables, and 
beyond with 64 bit executables. This puppy can run on virtually any computer; see, the below table of running times on a variety of computers.

\section{TART2012 DVD}

TART2012 is distributed on DVD. This DVD contains on-line documentation for all codes included in the system, the codes configured to run on a variety of computers, and many example problems that you can use to familiarize yourself with the system.

\section{TART Home Page}

Since I retired there is no longer an up-to-date TART Home Page at Lawrence Livermore National Laboratory. The BEST contact and source of information, reports and announcement is my personal e.mail address and website [12].

TART documentation is now available on the TART2012 DVD; it is currently not available on-line. But TART users should periodically check the website [12] for the latest news and on-line TART tutorials that are designed to aid TART users

\section{TART Hot Line}

Well, not exactly a hot line, but at least a place to turn to when you need help. If you have any difficulties setting up TART input, running it, or analyzing output, you can contact me at home. Although I am retired I am willing to try to help TART users. But please be aware that this will be strictly on my own time and not related in anyway to my previous employment at LLNL, so please keep your requests within reason. To contact me use [12]

\section{Background: Legacy, Schmegacy}

Because TART has a history of over forty years one might think that today's TART code is an old legacy code that is very much out-of-date and is teetering on its last legs. Nothing could be further from the truth. Today's TART code has been completely rewritten in the last few years, and is now one of the most modern, up-to-date Monte Carlo radiation transport codes available. All that remains of the earlier code, TARTND, is the user input and output interface, and the many hundreds of man-years of experience accumulated in using TART over the last forty years.

The earlier TARTND code was written in the LRLTRAN language (unique to Livermore), which limited the code for use only on Livermore's CRAY computers. TARTND was basically a high energy neutron and photon transport code. It was limited to high energy neutrons because it used unshielded, multi-group cross sections, and as such did not account for resonance self-shielding, and it had a very limited thermal scattering treatment, and only a hand full of groups at low energy; none below a milli-eV. In addition its use of Livermore's Evaluated Nuclear Data Library (ENDL) for neutron data further restricted TARTND to high energy neutron problems; as the author of ENDL, Robert J. Howerton stated [10] that ENDL was never intended for use in slow neutron 
applications, and gives poor results if it is misused in slow neutron applications. In addition TARTND was designed to run on the small computers that were available decades ago, e.g., geometry that could be modeled only in fairly limited detail.

In contrast today's TART code is written in such standard FORTRAN that it runs on everything from large central computers, to UNIX workstations, to MACs and PCs, using Windows or LINUX - like I said: this puppy runs on any computer. It also includes the multi-band method to handle resonance self-shielding [7], and a very accuracy and efficient method to handle free atom scattering [4], as well as thermal scattering law data to handle bound atom scattering [11]. Added with TART2005 is continuous energy cross sections. Whereas the old TARTND code used 176 neutron energy groups, mostly clustered at high energy, today's TART code uses 700 neutron energy groups, 50 per energy decade, uniformly spaced in the log of the neutron energy between $10^{-5} \mathrm{eV}$ and 1 $\mathrm{GeV}$. Compared to the hand full of low energy groups used by TARTND, today's TART code has 250 groups below $1 \mathrm{eV}$. In addition TART now uses the most up-to-date evaluated nuclear data, namely the ENDF/B-VII.1 [5]. It also uses the most modern evaluated photon interaction data, EPDL97 [6], which is now the official ENDF/B-VII photon interaction data library. In addition today's code is designed to run on today's computers - and tomorrow's computers; there is now no limit on any input parameters, e.g., geometry can be modeled using as much detail as needed, be it using one spatial zone, or millions of spatial zones, TART2005 can efficiently handle both extremes. In an attempt to try and make your life simpler when you do include more geometric details, you will find that TART2005 now includes many more input options that can simplify how you define geometry (note, that the geometry included with TART2005 is so general that no additional geometry were added for TART2012).

One thing that today's code has managed to carryover from the older TARTND code, is incredible speed. If you compare TART to other Monte Carlo radiation transport codes you will be amazed at how much faster TART is. But be assured that this speed is not accomplished by compromising accuracy; we have always used Howerton's first theorem, "We are in no rush for the wrong answer". The combination of speed and accuracy is achieved by using all appropriate methods that we learned in graduate school and beyond. For example, where other codes may ignore everything we learned and try to solve transport problems starting from basic principles, TART uses very efficient and accurate methods to account for resonance self-shielding, bound and free atom scattering, and an assortment of nuclear and atomic models, that allow transport calculations to be accelerated with no loss in accuracy. Indeed it is fair to says that over a given, fixed period of running time TART's results are more accurate than other codes that start from basic principles, and as a consequence these other codes can end up converging extremely slowly, and when they do eventually converge it is usually to the TART answer that you can obtain much sooner. 


\section{Continuous Energy Cross Sections}

With today's TART2005 the user has the choice to use: 1) multi-group, unshielded cross sections, or 2) multi-band cross sections to account for self-shielding, or 3) the new (2005) continuous energy cross sections (no groups), that include all of the details of cross sections. The choice can be used as a teaching tool, to determine the importance of multi-group and self-shielding for your applications, or if you wish, to minimize running time. It is not recommended that you try to minimize running time at the expense of accuracy, but for quick, initial calculations users may decide to use this option. The running time using these various representations of the cross sections are roughly in the ratio: 1 ) multi-group $=1,2$ ) multi-band $=1.3,3$ ) continuous energy $=2$, e.g., the difference in running time between multi-group and continuous energy is only a factor of 2 , so there is not a great deal of benefit to using anything except continuous energy cross sections; but the choice is yours.

This combination of a modern up-to-date code that runs on any computer, and the best available nuclear models, and nuclear and atomic data, makes today's TART system hard to beat for performance speed and accuracy. The transition from the older TARTND code to today's TART2005 code did not take place overnight; basically this transition began years ago with the release of TART95.

The original TARTND code has been used and distributed from Lawrence Livermore National Laboratory for many years, but was always limited because it was written in the Livermore LLLTRAN language, and designed only to run on CRAY computers. TART95, released in July 1995, was the first version of the code written in very conservative standard FORTRAN, designed to be used on virtually any computer. Subsequent release of TART96, TART97, TART2000 and TART2002 were designed to extend the general utility of the code to more areas of application, by concentrating on improving the physics used by the code, particularly with respect to newer neutron data, and more detailed neutron data. TART2000 and TART2002 further improved the physics, particularly with respect to newer photon data, and more detailed photon data [9]. TART2005 is a major step forward in terms of generalizing the nuclear and atomic data used, and greatly expanding the options available to define geometry. TART2005's new continuous energy cross sections and input options, and greatly improved consistency checking are designed to make the code more user friendly and to improve the reliability of results.

TART2005 completely supersedes all older versions of TART, and it is strongly recommended that users only use the most recent version of TART2005 and its data files. Today the recommendation is to use TART2012.

For a historical review of the improvements in TART since the release of the first computer independent version, TART95, see also the TART 2002 report [8d]. Here I only describe where we are today, in terms of currently available features, and I review the new conventions and input options; for complete details of input, see chapter 2 of this report on Input Parameters. 


\title{
Features of TART2005
}

\begin{abstract}
A COMPLETE SYSTEM: The TART system is not merely one single code to run Monte Carlo radiation transport calculations. It is a complete system, that help you check your input before you run calculations (this greatly improves reliability), simplifying running calculations (no complicated system dependent conventions to remember), and help you analyze results, by using interactive graphics, allowing you to overlay your results onto your geometry (this gives you the "big picture", that allows you to see the overall variation of energy deposition, flux...).
\end{abstract}

Part of this complete system is one, and only one, set of neutron and photon data for use in your applications. I have used my expertise to select what I consider to be the BEST data for use in applications, and this is what is supplied with TART. TART is designed as an application system, including the BEST code and BEST neutron and photon data that I can provide. There are any number of codes available that can be used to investigate the results based upon using one or another set of nuclear data; TART is not one of these codes. If you want to investigate the effect of using one cross section versus another I suggest you use some other code. Once you have verified that one set of cross sections is better than another, PLEASE, let me know, and I'll consider revising TART's data bases.

Basically if you are interested in neutron and/or photon applications, and obtaining the answer to your problems as accurately and as quickly as possible, then the TART system is designed for you.

COMPUTER INDEPENDENT: TART2005 today runs on every computer: large central computers, a variety of UNIX workstations (SUN, SGI, HP, DEC Alpha, Meiko, and IBM RISC), Power MACs and IBM PCs using Windows or LINUX. TART is written in such simple and standard FORTRAN that I feel confident to say that not only does TART run on all computers today, it will also run on new computers as they become available; so you can be sure that TART will be available for your use not only today, but also well into the future. Note, that today TART2012 has extended this computer independence to allow one single version of TART2012 to be used to produce 32 bit or 64 bit executables, for use on any computer.

It wasn't too long ago that Monte Carlo radiation transport codes could only be run on multi-million dollar central computers. Today my lap top computer allows me to take TART with me anywhere and to run enormous problems in a small fraction of the time it took on large central computers just a few years ago.

Not only is TART handy as far as its portability, you should also note the economic advantage of today's TART over the old TARTND code that only ran on CRAY computer. For example, my \$2,000, $1200 \mathrm{MHz}$ Laptop computer runs TART2005 over forty times as fast as TARTND on a multi-million dollar CRAY-YMP.

This speed is even further enhanced with TART2012; see the summary of the speed of TART at my website, http://home.comcast.net/ redcullen1/speed.htm. It is also 
interesting to note how far Personal Computers have come in the last few years. When TART95 was released in 1996 the then fastest available 486dx2/66 PC took 18,487 seconds to run a set of benchmark problems. Today's best time of 19 seconds is an incredible 973 times faster - this is due to advances in computer speed, compiler design, and TART design. Think about what this means. A problem that in 1996 took an entire 9 to 5,8 hour (480 minute) work day to complete, now takes LESS THAN 30 SECONDS!!!

The last point concerning computer independence that I'll mention relates to the many users who have asked me: isn't it more time consuming and inefficient to make TART so computer independent? He answer is, no it isn't. If anything being able to test TART using many different computer/compiler combinations makes my job easier, and makes TART far more reliable. Different compilers check for different possible errors or inconsistencies, so that I can find and fix as many problems as possible by using as many different computer/complier combinations as possible, making TART more reliable, and ultimately saving myself time and energy.

BEST OPTIONS USED AS DEFAULTS: In the past the users had to be aware of and set all sorts of options and insure they set them correctly in their input files. Now the defaults are what I consider to be the "BEST" set of options, so you need no longer be concerned with them in your input. These options include ALL energy limits for transporting and scoring neutrons and photons; these limits are now automatically set by the code to track and score over the entire energy range of the data in the neutron and photon data files. In addition the important options for resonance self-shielding, thermal scattering, and fluorescence, are initially to have these features turned on.

NEW INPUT OPTIONS: have been added to TART2005 to allow reactor noise analysis calculations (sentl 54 and 55), easier simulation of detectors (sentl 56), use either total or prompt nu-bar (sentl 57), and definition of zone volumes by user input (volume). Options have also been added to extend TART's capabilities for geometry, as well as to simplify and make input more user friendly. These options include: cubic and quartic (e.g., torus) surfaces, as well as macro "surfaces" xyzbox, xcan, xconic, and macro "volumes", xsurf, new rotation and spatial translation, surface cloning, new neutron and photon sources, see, Appendix D: Summary of New Conventions and Options. Note, that ALL of these input options were included in TART2005 and were found to be so general that no additional options were added for TART2012.

ENDF/B-VI NEUTRON AND PHOTON CROSS SECTIONS: Older versions of TART only used the Livermore ENDL library neutron data, which was primarily designed for use in high energy neutron applications. In contrast the ENDF/B-VI and VII neutron data is designed for general use at all energies [3]. Therefore using this data allows the code to be accurately used in a wider range of applications. TART2012 data was updated based on ENDF/B-VII.1.

TART2005 used ENDF/B-VI data, whereas currently TART2012 uses the ENDF/BVII.1 [5], as well as the EPDL97 photon interaction data, which has been adopted as 
the ENDF/B-VII standard [6].

If you are a fan of the older ENDL data, sorry, but this is no longer supported by TART. You should consider that most of the evaluations in ENDL were done by R.J. Howerton prior to his retirement in 1986 - let me repeat that - 1986 - 26 years ago. ENDL was great in its day, but compared to more modern evaluations, ENDL is now completely outof-date.

TEMPERATURE DEPENDENT NEUTRON DATA: In the past TART has always used nominally room temperature (293.6 Kelvin) neutron cross sections, and the standard distributed DVD still includes data only for room temperature. We can now prepare additional data files at virtually any temperature to meet programmatic needs [5]. PLEASE remember that I am now retired, so I cannot guarantee to meet your needs for data at other temperatures, but if you have a need please feel free to contact me and we can talk - but I cannot promise anything.

NEUTRON 700 GROUP TREATMENT: for cross sections over the energy range $10^{-5}$ $\mathrm{eV}$ up to $1 \mathrm{GeV}$. Older versions of the code used a 175 group treatment from $1.30910^{-3}$ $\mathrm{eV}$ up to $20 \mathrm{MeV}$, with most of the groups concentrated at higher energy; this limited accurate use of the code to higher energy applications. In contrast the current 700 group treatment is designed to accurately treat the entire neutron energy range, thereby allowing the code to be used for a wider range of applications. The 700 group structure is 50 groups per energy decade, equally spaced in the log of the energy, from $10^{-5} \mathrm{eV}$ up to 1 $\mathrm{GeV}$. As yet neutron data is only generally available up to $20 \mathrm{MeV}$, but as soon as higher energy data becomes available TART is ready to use it.

If you are a fan of the older 175 group treatment, sorry, but this no longer supported by TART. The objective is to continue to make TART useful in an ever wider spectrum of applications, and the limitation of the old 175 groups, which were intended only for use in high energy neutron applications, simply was incompatible with this objective.

PHOTON 701 POINT TREATMENT: for cross sections over the energy range $100 \mathrm{eV}$ up to $1 \mathrm{GeV}$. Older versions of the code used a 176 point treatment from $100 \mathrm{eV}$ up to 30 $\mathrm{MeV}$, with most of the points concentrated at higher energy; this limited accurate use of the code to higher energy applications. As with the 700 group neutron treatment, this new treatment of the photon cross sections is designed to accurately treat the entire energy range, allowing the code to be used for a wider range of applications. The 701 points is a fixed set of energy points, 100 points per energy decade, equally spaced in the log of the energy, from $100 \mathrm{eV}$ up to $1 \mathrm{GeV}$. Currently photon data is available up to $1 \mathrm{GeV}$, so that all 701 points are currently used by TART.

If you are a fan of the older 176 point photon treatment, sorry, but this no longer supported by TART.

IMPROVED NEUTRON FISSION TREATMENT: TART includes continuous energy sampling of fission spectra. Earlier versions of TART used equally probable 
energy bins; equally probable energy bins leads to rather large errors in sampling low probability ranges at low and high energy emission of neutrons. This is a win-win improvement in TART, where the sampling is now both more accurate and faster. TART also includes the option to use either the average number of neutrons emitted per fission, or to sample from the distribution of the number of neutrons emitted per fission (sentl 54). Also included in TART is an option to allow spontaneous fission sources (sentl 55). The combination of allowing spontaneous fission sources and sampling from the distribution of the number of neutrons emitted per fission, allows TART to be used to simulate reactor noise analysis problems involving neutron emission correlation effects. You now also have your choice to use either total nu-bar (prompt plus delayed), or prompt (ignoring delayed); this is controlled by sentl 57, with the default being total nubar. You can now include a separate energy and time dependence for delayed neutrons, also controlled by sentl 57. Appendix $\mathrm{C}$ illustrates the effect on reactivity of using total versus prompt nu-bar.

NEW UNRESOLVED RESONANCE REGION SELF-SHIELDING: TART includes an extension of the multiband self-shielding method [7] to the unresolved resonance region. See Appendix A for an illustration of the effect that this has on neutron cross sections. This unresolved resonance region treatment is used with the multi-band method as well as with continuous energy cross sections; this option is controlled by sentl 20 .

IMPROVED THERMAL SCATTERING TREATMENT: for both bound and free atom scattering. ENDF/B-VI thermal scattering law data is only available for a few materials [11]. TART now includes six of these in its neutron data library,

\begin{tabular}{|c|c|c|}
\hline$A=$ & 1801 & $\mathrm{H}$ bound in $\mathrm{H} 2 \mathrm{O}$ \\
\hline & 1901 & $\mathrm{H}$ bound in $\mathrm{CH} 2$ \\
\hline & 1902 & $\mathrm{D}$ bound in $\mathrm{D} 2 \mathrm{O}$ \\
\hline & 4809 & Be bound in Be metal \\
\hline & 4909 & $\mathrm{Be}$ bound in $\mathrm{BeO}$ \\
\hline & 6912 & $\mathrm{C}$ bound in graphite \\
\hline & 8916 & $\mathrm{O}$ bound in $\mathrm{BeO}$ \\
\hline
\end{tabular}

To use these in your applications you need merely specify the above ZA in place of the normal ZA when defining your materials in TART input. For example, for free $\mathrm{H}$ in $\mathrm{H} 2 \mathrm{O}$ the input is two parts $\mathrm{ZA}=1001(\mathrm{H})$ and one part $\mathrm{ZA}=8016(\mathrm{O})$; for bound $\mathrm{H}$ in $\mathrm{H} 2 \mathrm{O}$ you need merely specify two parts $\mathrm{ZA}=1801(\mathrm{H}$ bound in $\mathrm{H} 2 \mathrm{O})$ and one part $\mathrm{ZA}=8016$ (O). The except being that for bound $\mathrm{BeO}$ you must specify both $\mathrm{Z}=4909$ (Be bound in $\mathrm{BeO})$ and $\mathrm{ZA}=8916(\mathrm{O}$ bound in $\mathrm{BeO})$ - the above list includes seven items, but really only includes data for six materials, since for BeO both Be and O MUST be defined as bound.

Since thermal scattering law data is available only for a few materials, free atom scattering is still important, since it is used for all other materials. The major advantages of the new free atom thermal scattering treatment include: improved accuracy of sampling, and greatly improved speed of execution [4]. 
PHOTON SCATTERING TREATMENT: TART includes an improved treatment of photon coherent and incoherent scattering. The current treatment has the advantage that it is both more accurate, and faster to use, than the older treatment.

IMPROVED GEOMETRIC EXPERT SYSTEM: TART has always included an expert system that learns what your geometry looks like as your problem runs. This allows tracking through your geometry to accelerate during any given TART run. TART allows you to carry this learned information forward to successive TART runs. The information defining the connectivity of your geometry is automatically output to a file named GEOLINKS, and if you run a problem using the same geometry in the future, this information is read by TART, to allow it to accumulate experience and accelerate even further with each successive TART run.

IMPROVED NO UPPER OR LOWER LIMITS: on anything you can define by input. Unlike earlier versions of TART, that had a maximum allowed number of zones, surfaces, etc., today's TART has no limits at all. For example, earlier versions of the code were limited to a maximum of 1,000 spatial zones. Since then the spatial detail used in TART problems has increased enormously. The largest TART problem that I know of involved 27 million $(27,000,000)$ spatial zones. Of course TART can still accommodate even the simplest problem, such as a one zone spherical ball. In all cases from smallest to largest TART automatically sizes itself to accommodate each individual problem run. With the extension to 64 bit executables the number of spatial zones can be even further increased to meet virtually any need.

LONG RUN RANDOM NUMBER GENERATOR: The current random number generator includes over 2,500 different random number sequences, each sequence a trillion $\left(10^{12}\right)$ random numbers displaced from the preceding sequence. With a modern computer we can generate a trillion random numbers in about one day, if that's all a code is doing. With TART each random number sequence should take about 10 to 20 days to use one complete trillion number sequence. Therefore the currently available 2,500 sequences should keep you busy for years; and if you need more, just ask for them.

MULTIPROCESSING: The TART utility codes MULTIPRO and TARTSUM are now routinely used to perform multiprocessing. This approach to multiprocessing is so simple, straightforward and general that virtually all TART users can use it. With this approach if you have a computer with thousands of processors you can use MULTIPRO to create everything that you need to use as many processors as you want and then average the results together using TARTSUM. Even if you don't have a computer with many processors, but you do have access to a number of computers, you can use all available computers to run problems (they don't even have to be the same type of computer), and again use TARTSUM to average all of the results together. This approach is completely computer independent, and in the example case of using 250 processors, you can compress 250 days of work into a single day (more than a year of working days into one day). With the new random number generator, using different random number sequences for each run, you can make over 2,500 statistically independent runs and combine the results. 
Unlike the approaches used by other codes that are tied to specific computers, and have overhead due to communications between processors, with TART's approach you can use as many processors as are available, all at the same time, or at different time, and there is no overhead due to communications between processors, since each run is independent and contributes to the final results. You can do this simply by running the same problem with different random number sequences, either using multiprocessing, or any number of single processor computers that you have access to, or a single processor repeatedly, if you just want to run more histories to improve your results. This gives you the ultimate flexibility as far as how to best use the computer resources available to you.

IMPROVED INPUT CHECKING: to catch more input errors before the calculation begins. As described below, this checking is now incorporated in both TART and TARTCHEK. TART continues the TART traditions to support all older TART input parameters. For example, if you have a twenty year old TART input problem, you will still be able to use it today with TART. However, TART and TARTCHEK are now much cleverer at finding errors in TART input, with the result that you may find that TART input decks that ran earlier, will now cause TART to stop, with detailed ERROR messages asking you to correct your input before proceeding.

MODULAR CODING: TART is also very modular, so that portions of the code can be used in other codes. For example, TART and TARTCHEK use exactly the same input and geometry package; this assures that when you use TARTCHEK to check your geometry, when you run TART it will interpret your geometry in exactly the same manner.

INTERNAL CONSISTENCY CHECKING: No code is perfect, and for any complicated code, such as TART, that has many possible paths through it, it is virtually impossible to manually check all possible paths. The code now includes internal consistency checking; it does its own checking every time it runs. For example, every single array in the code is checked for misuse, in an attempt to find as many errors as possible. You wouldn't believe how effective this internal checking has been over the last few years at finding and allowing us to eliminate potential problems, resulting in a much more reliable code. This internal consistency checking has been so effective that since the release of TART98 not one single internal consistency problem has occurred. However, I have left the internal consistency checking in place within the code, so that any changes or new additions to the code are automatically verified.

FULL OPTIMIZATION: One general improvement worth noting, is that based on communications with a variety of FORTRAN compiler designers, TART's has been redesigned to allow it to be compiled at the highest level of optimization on most computers, which can greatly reduce running time, without sacrificing accuracy; indeed by understanding how the optimizers are designed, I have made TART much more reliable as far as its coding not being misinterpreted by compiler optimizers.

CONTINUATION OF ANY INPUT LINE: Any input line can now be continued onto any number of continuation lines. With earlier versions of TART some input, particularly 
complicated sources, could not be continued from one line to another, which made input preparation difficult. You will find that being able to continue any input line, it is much easier to prepare input. Some of the following new options, such as cloning, rotation and spatial translation, were recommended by TART users, and are also designed to simplify preparation of TART input. If you have ideas to even further simplify input preparation, I'd love to hear them.

GENERAL IMPROVEMENTS: TART is based on the older TARTND code, but required massive changes to the code to make it the modern, computer independent code that it is today. As such there were bound to be some growing pains with this essentially new code. Over the last few years, feedback from the many code users has led to general improvements in the code, both in terms of locating and correcting problem areas, as well as in adding and improving code options to meet the needs of users. Examples of overall corrections and improvements include,

1) Corrected Be-9 (n,2n) neutron emission spectra, which were incorrect.

2) Corrected $Z$ cone Reflection.

3) Corrected addxyz input.

4) Added zone volume user input option.

5) Added the option with tally types 11 and 12, to tally in zone neutron absorption, rather than every time a neutron enters a zone.

6) Allowed type 11 and 12 for empty (no material assigned) zones.

7) Added improved reflected calculations.

EXPERIENCE: Lastly I will mention is the other important change is EXPERIENCE!!! Again, I cannot stress how important this is for any code. In the case of TART each successive version of the code includes the operating experience of the many people who are now using the code. With each passing version of TART reliability and accuracy are improved, mostly based on feedback from users - such as you - so keep that feedback coming. Even though I am retired I do try to keep us with user feedback. 


\section{Running Time}

The below table presents results obtained using a collection of 68 TART benchmark problems. All 68 problems were run on each computer. This table summarizes timing results for the older TARTND code that only runs on CRAY computers, as well as TART 2002, or TART2005, and TART95 on a variety of computers. This list is from the TART2005 report, and is now somewhat out of date, but will help to give first time readers of this report some idea of the advances that we have seen over the years. For the most up-to-date list of running times see, http://home.comcast.net/ redcullen1/speed.htm

\begin{tabular}{|c|c|c|c|}
\hline Code & Computer & $\begin{array}{l}\text { Running } \\
\text { Time } \\
\text { (Seconds) }\end{array}$ & $\begin{array}{l}\text { Ratio to } \\
\text { TARTNP } \\
\text { CRAY-YMP }\end{array}$ \\
\hline TARTNP & CRAY-YMP & 5396 & 1.0 \\
\hline TARTNP & CRAY-J90 & 7727 & 1.43 \\
\hline TART2005 & AMD $3500+$ & 47 & 0.0087 \\
\hline TART2005 & AMD $3400+$ & 48 & 0.0089 \\
\hline TART2005 & IBM-PC Pentium IV/3600 & 58 & 0.0107 \\
\hline TART 2002 & Athlon XP1800/1520 & 89 & 0.0165 \\
\hline TART 2002 & IBM-PC Pentium IV/2000 & 132 & 0.025 \\
\hline TART 2002 & IBM-PC Lap Top III/1200 & 133 & 0.025 \\
\hline TART 2002 & IBM-PC Pentium III/1000 & 170 & 0.031 \\
\hline TART 2002 & IBM-PC Pentium III/500 & 500 & 0.09 \\
\hline TART 2002 & DEC-Alpha Model 5/625 & 516 & 0.10 \\
\hline TART 2002 & IBM-PC Pentium II/400 & 579 & 0.11 \\
\hline TART 2002 & PowerMAC/LapTop/500 & 683 & 0.126 \\
\hline TART 2002 & IBM-PC Pentium II/333 & 697 & 0.13 \\
\hline TART 2002 & DEC-Alpha Model 5/300 & 712 & 0.13 \\
\hline TART 2002 & IBM-PC Pentium II/266 & 855 & 0.16 \\
\hline TART 2002 & IBM-PC Pentium Pro/200 & 1185 & 0.22 \\
\hline TART 2002 & IBM-PC Lap Top/233 & 1301 & 0.24 \\
\hline TART 2002 & Power-MAC $7500 / 275$ & 1350 & 0.25 \\
\hline TART 2002 & iMAC & 1664 & 0.31 \\
\hline TART 2002 & $\mathrm{HP}-735 / 125$ & 1834 & 0.34 \\
\hline TART 2002 & SUN E3000/166 & 2107 & 0.39 \\
\hline TART 2002 & IBM-PC LapTop/133 & 2990 & 0.58 \\
\hline TART 2002 & CRAY-YMP & 4262 & 0.79 \\
\hline TART 2002 & IBM-RISC RS-6000 & 5739 & 1.06 \\
\hline TART 2002 & CRAY-J90 & 6095 & 1.13 \\
\hline TART 2002 & Meiko CS-2/66 & 6225 & 1.15 \\
\hline TART 2002 & SUN Sparc-20 & 6315 & 1.17 \\
\hline TART 2002 & Power-MAC 7500/100 & 6446 & 1.21 \\
\hline TART 2002 & SGI R4000/100 & 6953 & 1.29 \\
\hline TART 95 & CRAY-YMP & 4912 & 0.91 \\
\hline TART95 & $\mathrm{HP}-350$ & 4322 & 0.80 \\
\hline TART 95 & DEC-Alpha & 6130 & 1.14 \\
\hline TART95 & SUN & 9673 & 1.79 \\
\hline TART95 & Meiko & 9993 & 1.85 \\
\hline TART95 & SGI & 10157 & 1.88 \\
\hline TART95 & IBM-RSIC & 14838 & 2.75 \\
\hline TART 95 & IBM-PC $486 \mathrm{DX} 2 / 66$ & 18437 & 3.41 \\
\hline
\end{tabular}

When we compare the codes all run on the same CRAY-YMP, we find that compared to the older TARTND code, TART95 was about $9 \%$ faster, TART 2002 was about $21 \%$ faster, and even though TART2005 has not been run on a CRAY-YMP its scaling on other computers indicates that it is even faster than TART 2002. So that not only has TART been extended for more general uses, these extensions were accomplished with no 
lose in running time efficiency, with each successive release of TART being even faster than the preceding version.

You should also note the advantage of TART2005 over the older TARTND in terms of their ability to be used on virtually any computer. For example, a $\$ 2,000$ Pentium III, $1200 \mathrm{MHz}$ Laptop computer runs TART 2002 over forty times as fast as TARTND on a CRAY-YMP, and the Pentium-IV, $3600 \mathrm{MHz}$ computer runs TART2005 over ninety times faster, and on an AMD 3500+ over a hundred times faster.

\section{Why is Monte Carlo Used so much Today?}

The last point to note from these comparisons is how far we have come in terms of available inexpensive computer power in the few years between the release of TART95 and TART2005. When TART95 was released the fastest IBM-PC then available took 18,437 seconds to run this collection of 68 problems. Even then we could foresee the potential of an inexpensive computer being able to run these problems in only about 3.4 as much time as it took on a CRAY-YMP. But I don't think anyone could foresee that just a few years later we would have available IBM-PCs that can run this collection of problems in only 58 seconds. Compared to the PCs of only a few years ago, not only does today's PC run these problems over $\mathbf{3 1 7}$ times faster, but also it does it at about half the cost.

Think about what a difference in running time of a factor of $\mathbf{3 1 7}$ means. A major expense of any scientific project is your salary, so time is money and it can be expensive - or inexpensive, depending on how you spend it. Consider that only a few years ago if it took an entire 9 to 5,8 hour working day (480 minutes), to run a TART problem on an IBMPC, today it would take less than one minute ( 58 seconds) to run the same problem, i.e., a factor of 317 faster. It should be noted, that this tremendous increase in available inexpensive computer power is one of the reasons that the use of Monte Carlo has expanded so much in recent years. Problems that we thought too time consuming to be practical just a few years ago, have now become routine.

This speed is even further enhanced with TART2012; see the summary of the speed of TART at my website, http://home.comcast.net/ redcullen1/speed.htm. It is also interesting to note how far Personal Computers have come in the last few years. When TART95 was released in 1996 the then fastest available 486dx2/66 PC took 18,487 seconds to run a set of benchmark problems. Today's best time of 19 seconds is an incredible 973 times faster - this is due to advances in computer speed, compiler design, and TART design. Think about what this means. A problem that in 1996 took an entire 9 to 5,8 hour (480 minute) work day to complete, now takes LESS THAN 30 SECONDS!!! 


\section{Why is TART so FAST?}

Some users make the mistake of assuming that since TART is so much faster than other codes that perform the same types of calculations, the results based on other codes must be better than those based on TART. When you use TART you will find that its results are just as accurate as those of other codes, and often more accurate. So why is TART so fast?

There isn't any big secret to TART's speed: TART includes the three most important things necessary for generally efficient and accurate programming:

\section{EXPERIENCE! EXPERIENCE! EXPERIENCE!}

It is as simple as that. TART is based on almost 50 years of continuous use and improvement. During this time over a hundred work years of physicist/programmer time, and many hundreds of work years of user experience, were incorporated into the code that we have today. To illustrate why TART is so much faster than and still as accurate as other codes, I'll mention just a few points.

First is the use of multi-group data, including the multi-band method to account for selfshielding $[1,7]$, as used by TART, compared to continuous energy cross sections used to other codes. Results using continuous energy cross sections have to be better, right? Not always! This is only true if you run a calculation for extremely long times so that you accurately sample ALL of the continuous energy cross sections. This is almost never done, and I know of no code that explicitly includes an estimate of the uncertainty in its results based on the enormous variation in continuous energy cross sections. In comparison, TART's approach is designed for the real world, and incorporates not only the best nuclear and atomic data, but also the best nuclear and atomic engineering.

There is a reason that we went to graduate school and studied nuclear engineering and physics, and all of the methods and experience gained through decades and decades of real World experience. I have tried to incorporate all of this knowledge into TART so that users can obtain the most accurate answers in the least amount of time. Sorry to say that some other similar codes have been written starting from basic principles which sounds good, but in reality this approach can end up failing to use everything we were taught in school, and costing you the code user both time and accuracy. 


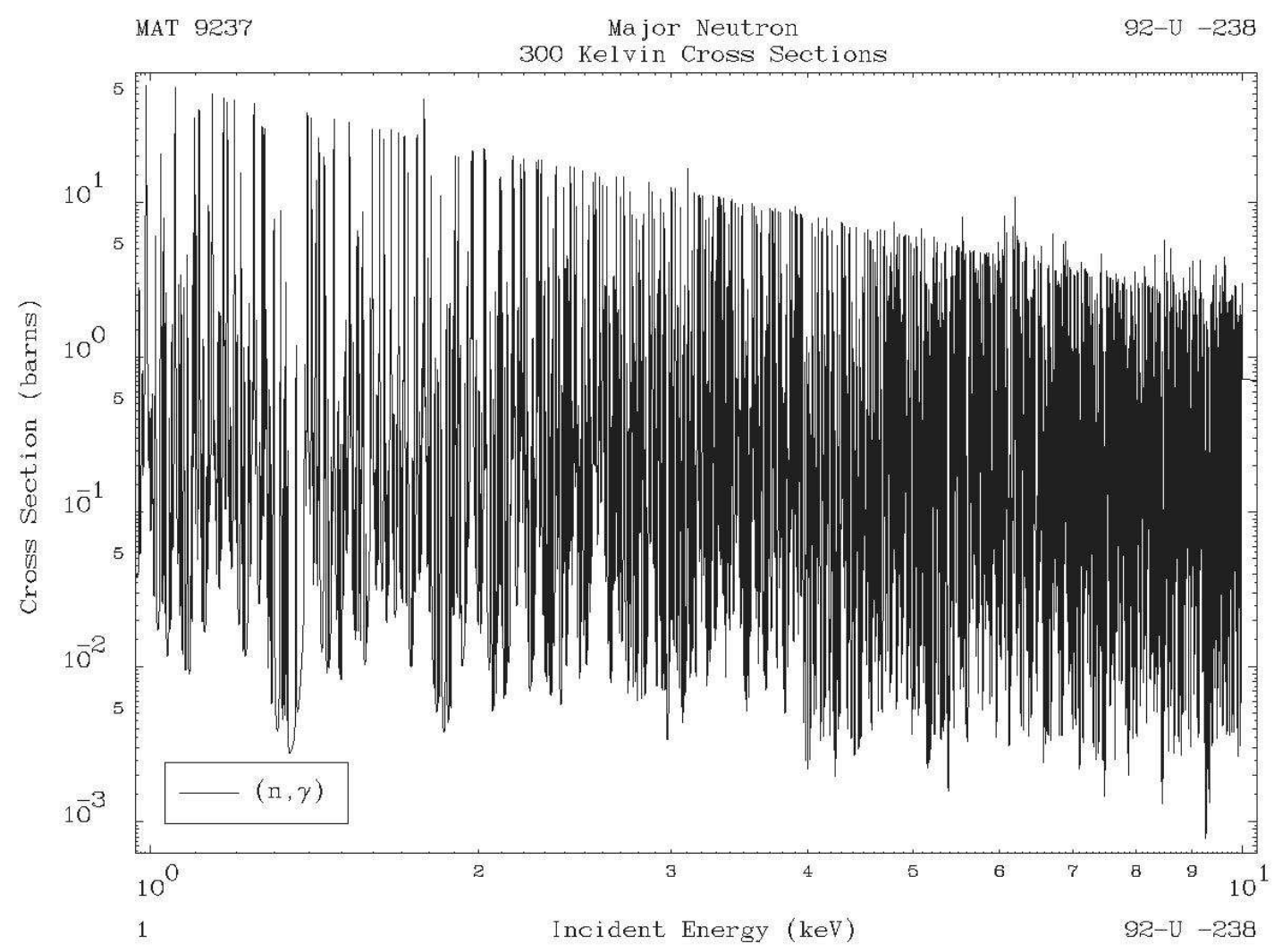

For example, if we look at the U-238 cross sections we see capture cross sections even over a fairly small energy range of 1 to $10 \mathrm{keV}$, that vary by roughly four orders of magnitude, and we can see that it is composed of very narrow resonances with relatively large energy intervals between resonances, i.e., the ratio of resonance spacing to width is about 100 to 1 . This data is VERY DIFFICULT to sample on a continuous energy basis. Indeed if you try it you will find that in order to obtain even a fairly accurate estimate of the average cross sections and distance to collision you would have to sample billions of histories. I don't know of any code that uses continuous energy cross sections that actually does this. They simply supply you with the "best" possible cross sections and assume that this will solve your problems.

TART takes it a step further: not only does TART use the "best" cross sections, but also uses the "best" nuclear engineering. Again, consider the U-238 cross sections. Anyone who has taken a course in reactor physics knows that in this case the neutron flux will self-shield and we know the form of the self-shielding. Therefore we do not need all of the nitty-gritty details of each and every narrow capture resonance in order to perform an accurate transport calculation. Think about it: people have been successfully designing nuclear reactors for over 60 years, and yet only fairly recently have detailed cross sections become available. So how did people design their reactors? They did what TART now does: combine the "best" currently available nuclear data with the "best" nuclear reactor theory. In the case of TART the use of the multi-band method to account for resonance self-shielding $[1,7]$ allows it to use multi-group, rather than continuous energy 
cross sections, resulting in rapid convergence of calculations, compared to code that use continuous energy cross sections and take forever to converge. Most important for users to understand is that this is done with virtually no lose in accuracy in the TART calculations, indeed it is fair to say that since for reasonable running times the TART results converge and those of other codes do not, from the pragmatic viewpoint of obtaining accurate answers in a reasonable amount of time, the TART results are better.

I should also mention the unresolved resonance region, where by definition we do not know the cross sections on a continuous energy basis, but it can be accurately treated by the multi-band method used by TART. Ask yourself: what do codes that claim to use continuous energy cross sections do in the unresolved resonance region? Only a few Monte Carlo codes try to account for self-shielding in the unresolved energy region. Who knows what other codes use.

However, if you are not convinced that the multi-band method is fast and accurate for your applications, you now have the option to use continuous energy cross sections with TART; this is controlled by sentl 20. After implementing the continuous energy treatment in TART and testing it for speed and accuracy in many applications, my conclusion is that as yet I have not found any application where the multi-band method seriously fails. Indeed as yet the major use that I have found for the continuous energy cross section treatment is to directly verify the accuracy of the multi-band method.

I should mention here that there is not that big a difference in running time using multiband or continuous energy cross sections (roughly a ratio of 1 to 1.6 in running time); so there is not a big penalty in using continuous energy cross sections. But carefully check the final uncertainty on your results. The ratio 1 to 1.6 applies to running the same number of source neutrons; you may require more source neutrons when using continuous energy cross sections, because of the additional statistical uncertainty introduced by large variations in the cross sections.

A second example of why TART is so fast is its treatment of geometry. Compared to other codes TART uses a very strict geometry, which places an additional burden on the user in terms of input preparation. But the pay off is that the input is easier to check and correct (using TARTCHEK) to improve reliability, and when the code starts to run it FLIES!!!

For example, TART insists that the users define every space point to be within a spatial zone. Other codes do not insist on this, so why does TART? The first reason is that without insisting on this it is not possible to check the input parameters for errors; checking is now simple and straightforward using TARTCHEK, and greatly improves the reliability of the input. Next, when TART runs it greatly accelerates tracking. How can a few holes in the geometry make such a big difference? Consider a simple problem involving 1000 spatial zones with each zone bounded by 6 surfaces. When a particle enters a spatial region that is not defined in the problem, i.e., is a "hole", the code has to track (ray trace) to the nearest bounding surface to determine what zone it will next enter. In this example it has to ray trace to the 6 bounding surfaces of each of the 1,000 spatial zones, to determine which of these surfaces is closest to the particle in its direction of 
travel, i.e., it has to ray trace to 6,000 surfaces. In contrast, with TART where a particle is always within a defined zone, in this example, neutrons are always inside one of the zones and we only have to track (ray trace) to the nearest boundary of the zone. This only involving tracking to each of the bounding surfaces of this one zone, i.e., ray trace to 6 , rather than 6,000 surfaces. No wonder TART geometry is so much faster to track through. How much of an effect does this really make? TART and TARTCHEK use exactly the same geometry package. In the original method used by TARTCHEK to display 3-D objects, TARTCHEK used a general ray tracing technique that did not take advantage of TART geometry. When TARTCHEK was updated to take advantage of TART geometry the ray tracing to display 3-D objects ran up to 200 TIMES FASTER - not $200 \%-200$ TIMES (20,000\%)!!! Pictures that took hours or all night to produce could suddenly be done in minutes or seconds. The difference was dramatic. You can see for yourself; use TARTCHEK to display 3-D views of your geometry and you will be amazed at how fast it can do it - and remember in doing it, it is using EXACTLY the same routines that TART uses to track through 3-D geometry. No wonder TART is so fast.

These are but a few examples of why TART runs so much faster than other codes, with essentially no lose in accuracy. Try it for yourself and see what you think.

As related to reliability, I'll also mention in passing the danger of using the default of other codes that assume that whatever volume you have not explicitly defined is vacuum that the code can freely transport through. My experience has been that when a problem has an undefined volume in it, well over $90 \%$ of the time it is because it is an error. Other codes sweep this under the rug and make it appear that nothing is wrong, usually resulting in the wrong answer. In contrast TARTCHEK and TART will quickly find these volumes and ask you to explicitly define them. This approach greatly improves the reliability of the TART input. 


\section{What Code should you be using?}

TART2012 completely supersedes all older versions of TART, and it is strongly recommended that users only use the most recent version of TART2012 and its data files. Below I illustrate how to check the date of the code and its data files, using TART2005 as an example; you can easily do the same for TART2012.

How do you know if you have the most recent version of the code and its data files? As soon as the code starts to run it identifies the version you are running and the dates of its data files. Below is the beginning of the code output report. Note, the code version: TART05-4, Nov. 2005, and the date of the five data files is $\mathbf{0 5} / \mathbf{0 5} / \mathbf{0 5}$. Note, also the newer 616 groups for the neutron data and $\mathbf{7 0 1}$ points for the photon data. If you are using an older version of the code or its data files, it is strongly recommended that you obtain the most up-to-date code and data; see, the below section on Availability.

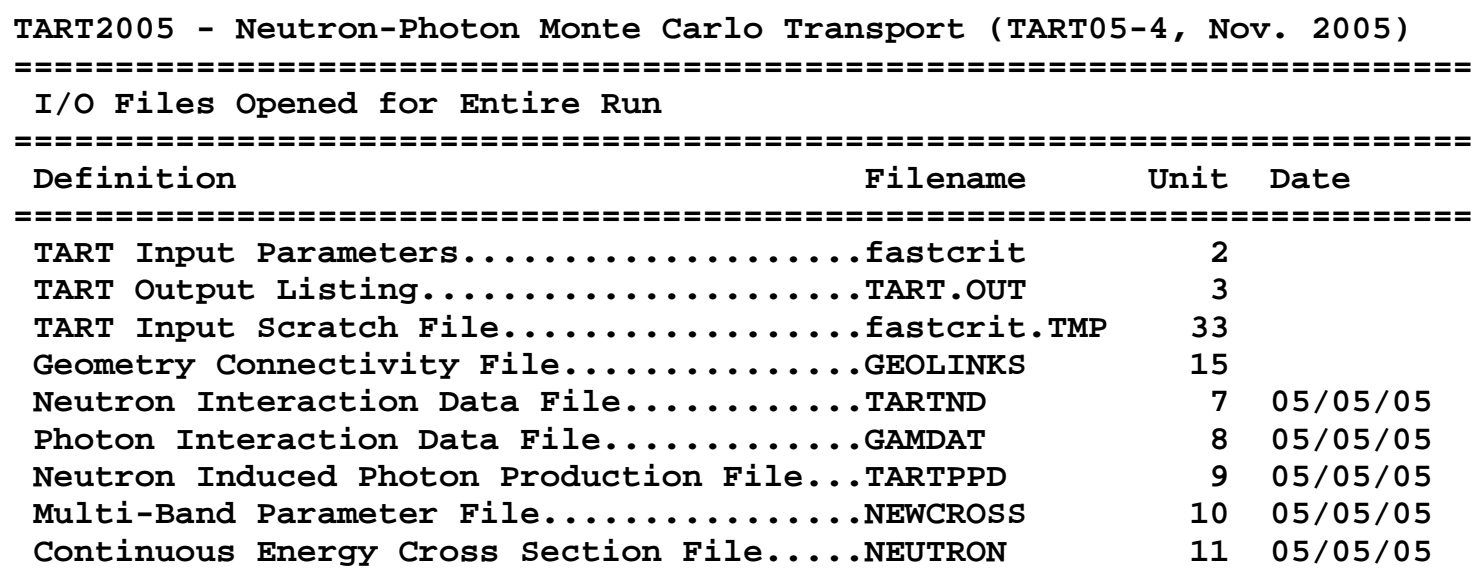

Neutron Interaction Data. 616 Groups $1.0000 \mathrm{D}-11$ to 2.0000D+01 MeV Photon Interaction Data.. 701 Points 1.0000D-04 to 1.0000D+03 MeV

\section{Utility Codes}

In addition to the TART code you should also be aware of the utility codes distributed with TART; of particular note are TARTCHEK and TARTAID. One of the most difficult tasks that you will face in using any 3-D combinatorial Monte Carlo code is to correctly define input parameters for the code, particularly to correctly define geometry. This is what TARTCHEK is designed to help you with. It is an interactive graphics code that will allow you to view and check your input parameters before you run TART. Even we so called "experts" on TART find that using TARTCHEK can greatly reduce the amount of time that we have to spend on input preparation, and even what is more important, greatly improve the reliability of our input parameters. In addition the TART2005 DVD system includes TARTAID, which will allow you to interactively create TART input decks from scratch.

By now most, if not all, TART users are familiar with TARTCHEK's ability to show 
you your geometry in 2-D, and to quickly test for error in your TART input parameters. But many users are still not familiar with two additional capabilities of TARTCHEK as shown below: first its ability to show you geometry in 3-D, and second its ability to overlay results of a TART calculation on your geometry. Particularly the latter is an extremely powerful tool to help you "see" the big picture, as far as global variations of energy deposition or flux. Instead of spending days or weeks wading your way through a thick output listing trying to understand the results, using TARTCHEK a few minutes after you finish a TART calculation you can "see" the results overlaid on your geometry. Not only will this save you time, it can improve your overall understanding of the results, by showing you the "big picture" of how flux, deposition. etc., in each zone is related to that in all other zones. This is something that is very difficult to "see" regardless of how long you stare at an output listing. If you are not using TARTCHEK you are only making your job more difficult, and you don't know what you are missing.

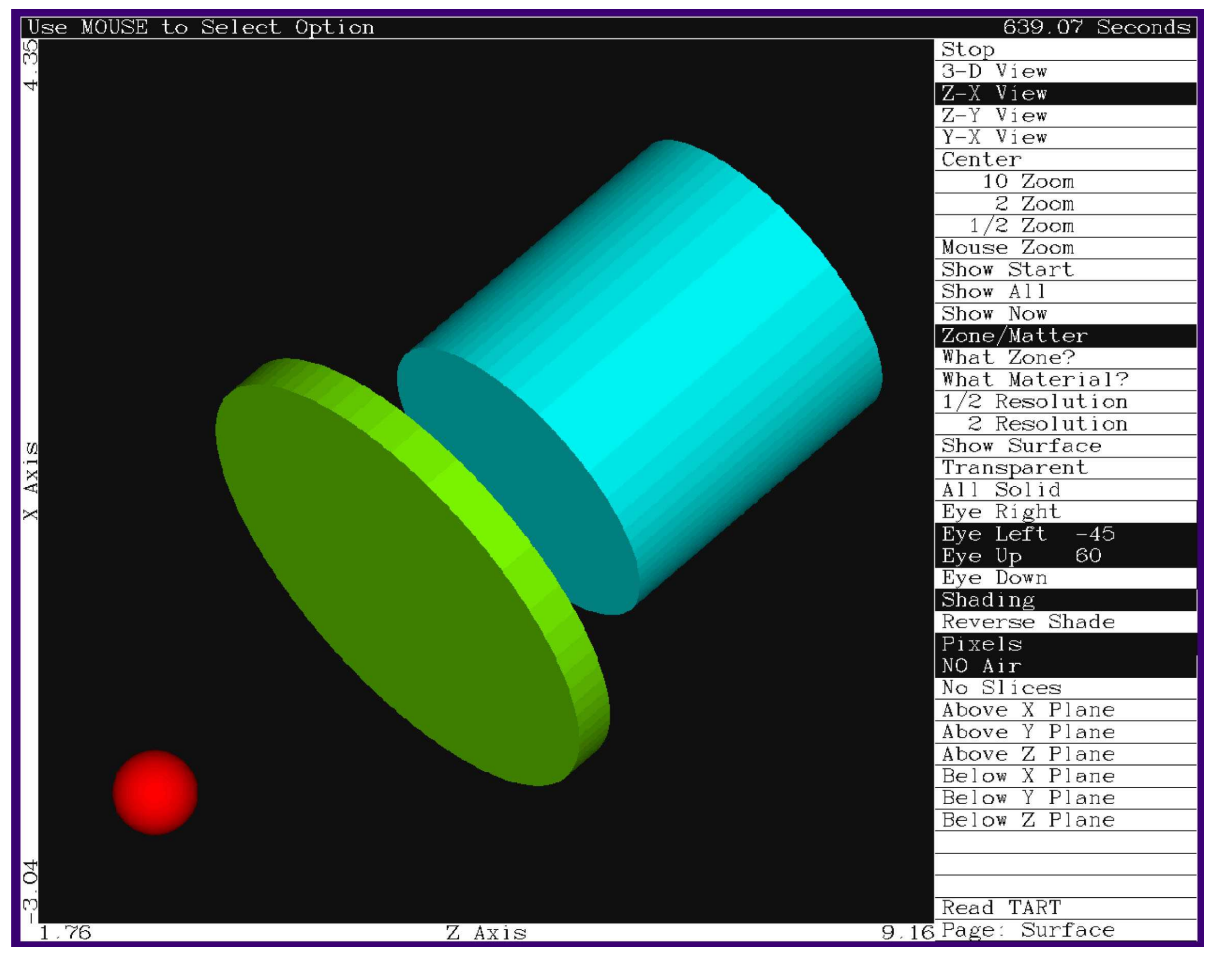

Example of 3-D view of geometry 


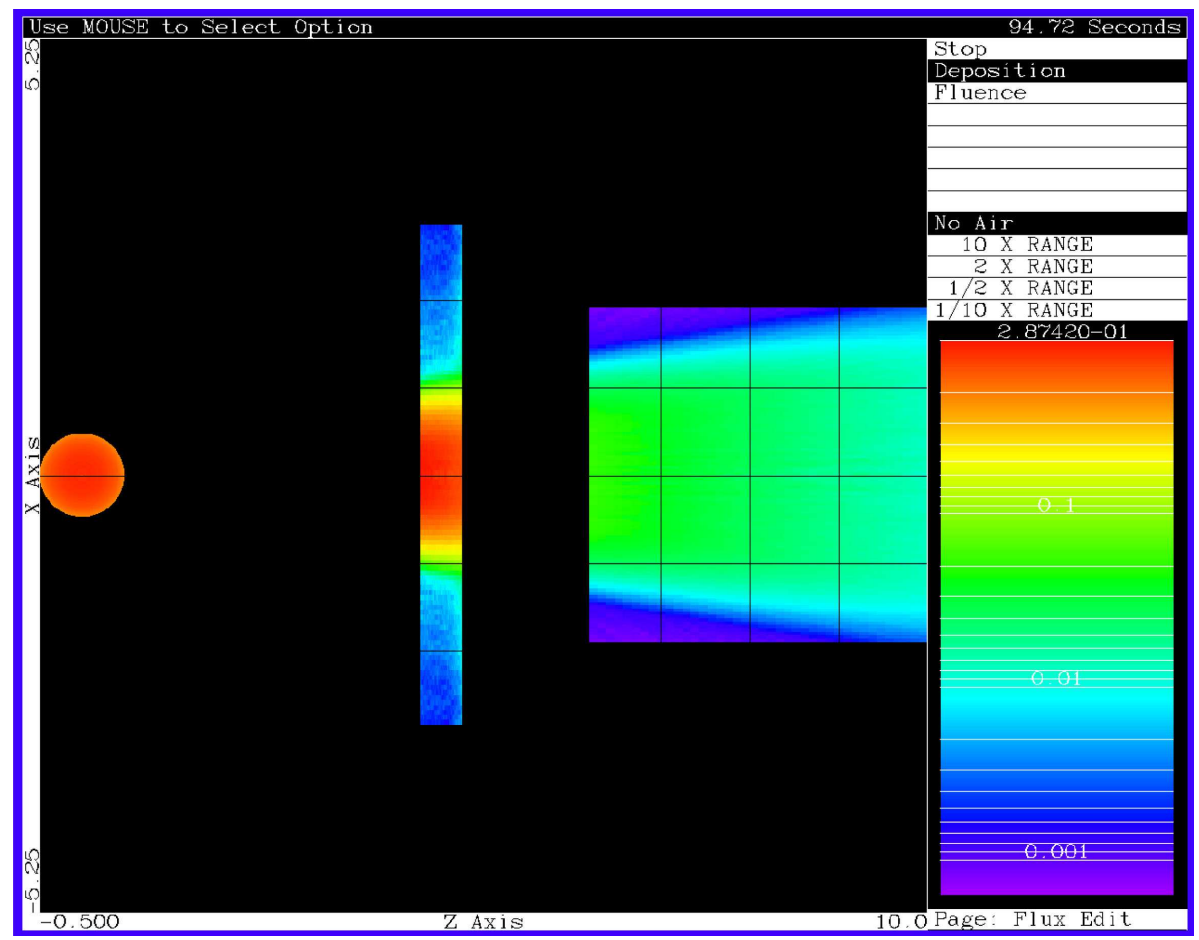

Example of energy deposit overlaid on geometry

TARTAID is another code you should be aware of. In addition to TARTCHEK, which can be used to check existing TART input, and display TART results, the TART2005 DVD system also includes TARTAID. This code is designed to help you create TART input from scratch. It is particularly helping to define very detailed geometry, involving many spatial zones. For example to create a TART input deck involving 10,000 or even 100,000 spatial zones, takes only minutes using TARTAID.

You should also be away of the utility codes MULTIPRO and TARTSUM, which will allow you to easily run many TART problems simultaneously, and then add together results from any number of TART problems, and produce a combined output file in EXACTLY the same format as any other single TART problem output file. Our computers are getting faster and faster, but we are running into the speed of light problem, where we can only get so much work done using a single processor. TART's approach to multiprocessing allows us to avoid this limit, in the sense that we can now compress the work that used to take many days, into a single day. This is true on either multiprocessing computers or a group of single processors computers. Just run your problems on ANY computer(s), using as many processors as you have access to, and TARTSUM will combine the results for you. Note, since the combined output file produced by TARTSUM is in EXACTLY the same format as any other single TART problem output file, if you are one of the many TART users who have utility codes to further process TART output results - not to worry - your utility codes will work on the combined file, exactly the same way they work on the results of a single TART run. 


\section{Documentation}

Although TART2005 and TART2012, supersede all earlier versions of TART, the most complete documentation for TART is still,

TART95: A Coupled Neutron-Photon Monte Carlo Transport Code, Lawrence Livermore National Laboratory, UCRL-MA-121319, July 4, 1995, by D. E. Cullen, A.L. Edwards and E.F. Plechaty

This document, as well as all other TART documentation, is now available on the TART2012 DVD.

\section{Availability}

In the past I personally distributed TART within LLNL. Currently since I am retired and no longer located on site, I can no longer offer this service to provide TART to users at LLNL, but Dave Heinrichs has kindly agreed to distribute TART2012 within LLNL.

At Livermore, for copies of the system, contact Dave Heinrichs, X-45679.

Outside of Livermore, within the United States, contact the Radiation Safety Information Computational Center (RSICC), Oak Ridge National Laboratory (e. mail: pdc@ornl.gov),

Outside of the United States, contact the OECD Nuclear Energy Agency/Data Bank (NEA/DB), Paris, France (e. mail: programs@ @ea.fr).

\section{Code Installation}

The code is distributed with detailed instructions concerning installation and testing of the code. These instructions are periodically updated for distribution with the code, to insure that the instructions are as up-to-date as possible, and exactly correspond to the version of the code that you will be implementing and using. As such, installation instructions will not be included here, i.e., see the TART2012 DVD that you receive for the most up-to-date instructions. 


\section{Appendix A: Illustration of Resonance Region Self-Shielding}

The below figure illustrates the effect of resonance self-shielding on the 700 group U-233 cross sections used by TART. 700 groups may seem like a lot and you might think that there would be little or no resonance self-shielding. If this were the case the self-shielding factors, which are defined as the ratio of self-shielded to unshielded cross section, would be unity in all groups. The below figure illustrates that this is not the case, because the 700 groups are not nearly enough to accurately represent the energy dependent shape of the many narrow resonances in U-233. In the case of U-233 the resolved resonance region extends up to $600 \mathrm{eV}$, and the unresolved resonance region extends from $600 \mathrm{eV}$ up to 30 $\mathrm{keV}$ (indicated by the two vertical lines on the below figure). Note, that the current TART data includes self-shielding in both the resolved and unresolved resonance regions. Note, we are not talking about small differences here; as we can see from the below figure many of the self-shielded group averages are 20 to $30 \%$ less than the unshielded values, and in one case only half $(50 \%)$.

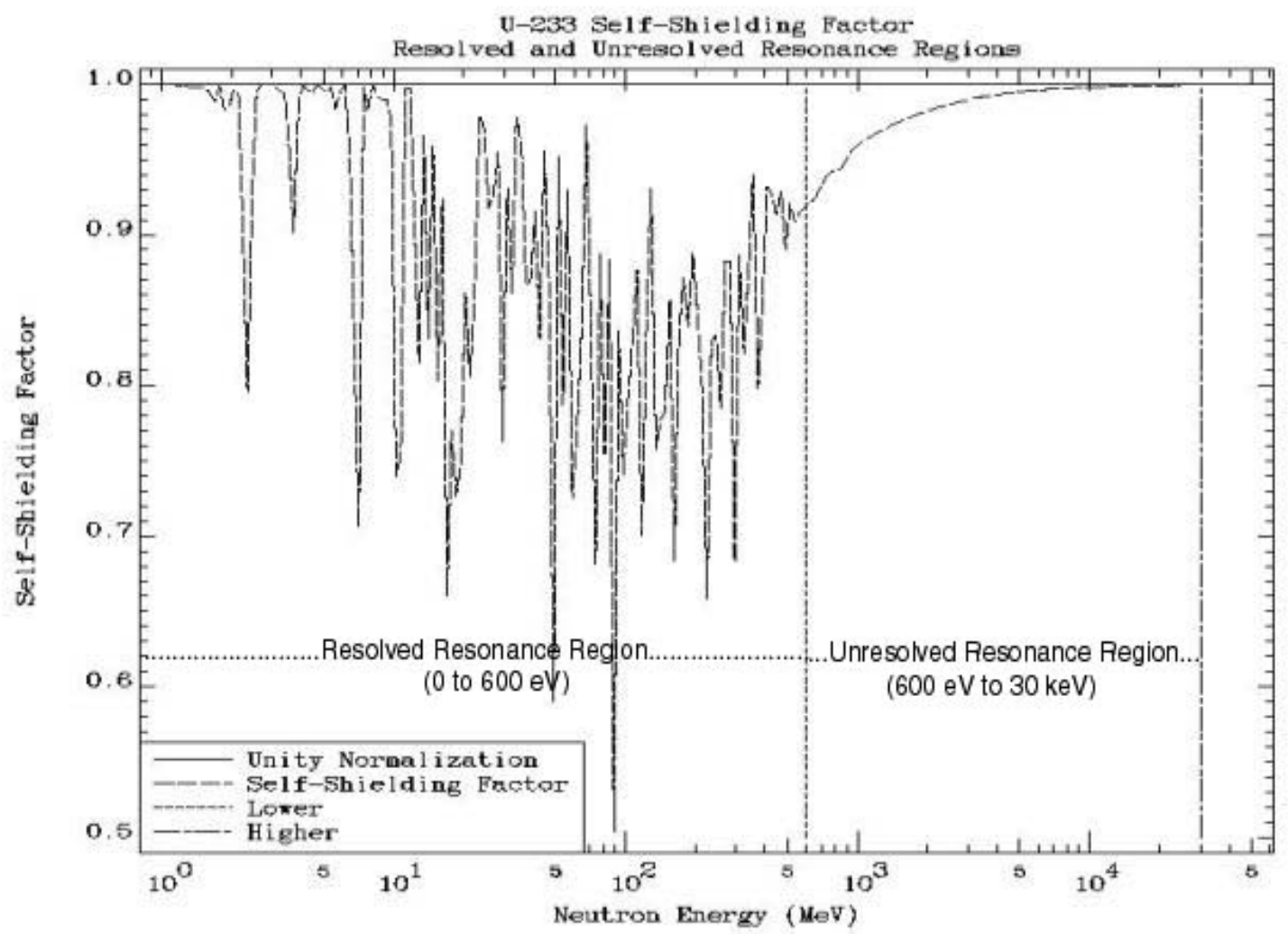

Example of self-shielding in U233 


\section{Appendix B: Comparison of Bound and Free Atom Be-9 Data}

The below figure illustrates the effect of molecular binding on the total cross section of Be-9. At higher energies, above the eV energy range, the effects of binding are negligible, and the cross sections are identical. At lower energies note the discontinuities in the bound cross at Bragg edges, and below about 5 milli-eV the bounding cross section deceases by over an order of magnitude.

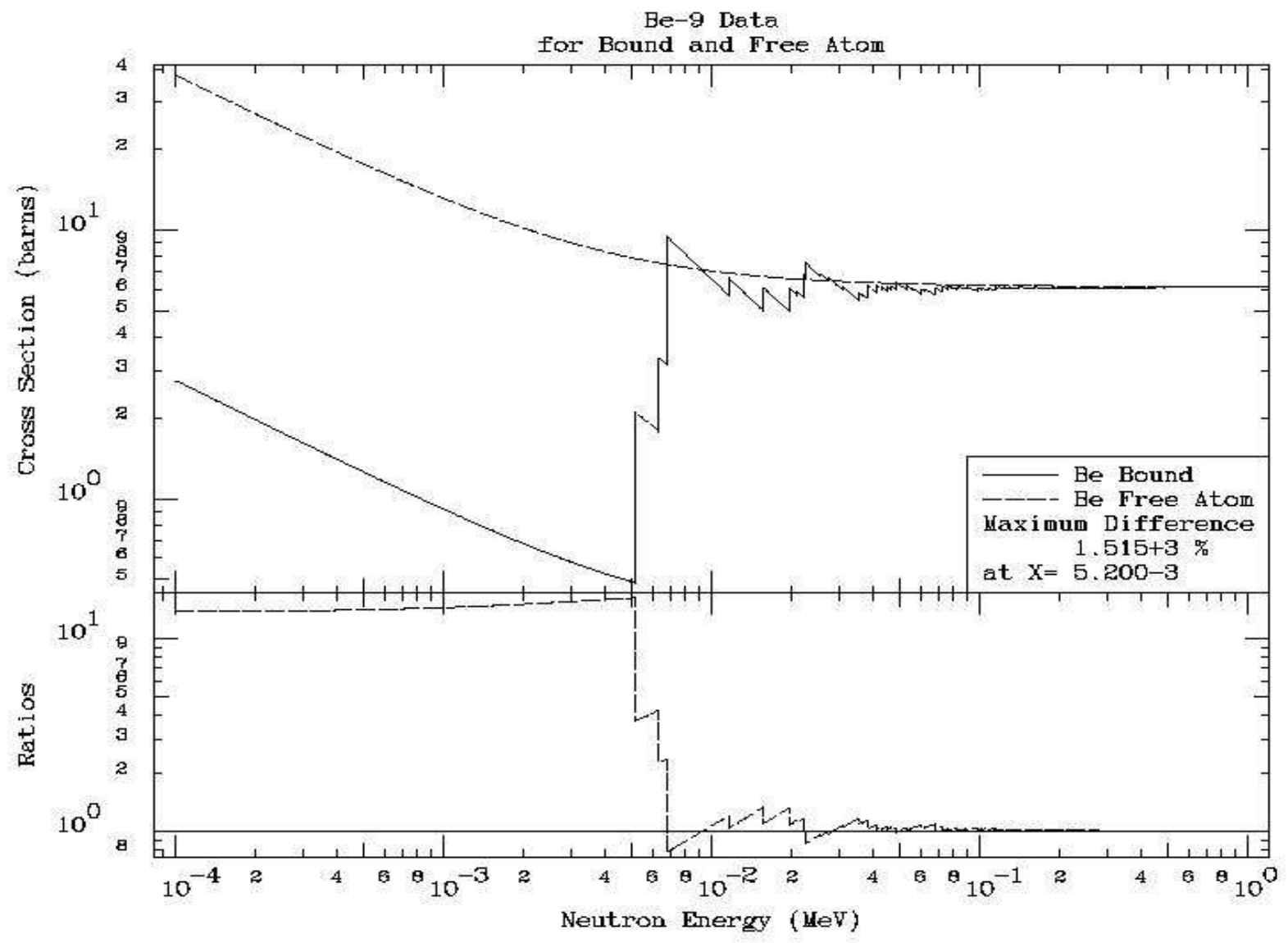




\section{Appendix C: The Effect on Reactivity using Total versus Prompt Nu- bar}

The below table illustrates the difference in reactivity (K-effective) of the 68 criticality problems distributed with TART using either total (prompt and delayed) or only prompt number of neutrons per fission (nu-bar). Note that the difference depends on the type of fissile material used, where the delayed fraction is small for $\mathrm{Pu}-239$, intermediate for $\mathrm{U}$ 233 , and larger for U-235, which can be seen by the magnitude of the differences in Keffective for these three different materials in a variety of systems.

Averaged over all 68 criticality problems the K-effective using only prompt neutrons is over $0.5 \%$ lower than when the total (prompt plus delayed) neutrons per fission (nu-bar) is used; prompt $=\mathbf{0 . 9 9 4 1 6 3}$, total $=\mathbf{0 . 9 9 9 3 4 0}$ (see, the end of the below table).

\begin{tabular}{|c|c|c|c|c|c|c|c|}
\hline Problem & Fuel & \multicolumn{2}{|c|}{ Reflector } & \multirow{2}{*}{$\begin{array}{l}\text { Total } \\
1.000290\end{array}$} & \multirow{2}{*}{$\begin{array}{l}\text { Prompt } \\
0.997982\end{array}$} & \multicolumn{2}{|c|}{ Difference } \\
\hline c10100 & $\mathrm{pu}-\mathrm{a}$ & be & 5.222 & & & -0.231 & $\%$ \\
\hline c20100 & $\mathrm{pu}-\mathrm{a}$ & be & 8.170 & 1.002780 & 1.000700 & -0.208 & $\%$ \\
\hline c30100 & $\mathrm{pu}-\mathrm{a}$ & be & 13.000 & 1.004140 & 1.002190 & -0.195 & $\%$ \\
\hline C40100 & $\mathrm{pu}-\mathrm{d}$ & & & 0.997953 & 0.995940 & -0.202 & $\%$ \\
\hline c50100 & $\mathrm{pu}-\mathrm{d}$ & be & 3.690 & 0.998140 & 0.996117 & -0.203 & $\%$ \\
\hline c60100 & $\mathrm{pu}-\mathrm{d}$ & be & 5.250 & 0.999445 & 0.997735 & -0.171 & $\%$ \\
\hline c70100 & $\mathrm{pu}-\mathrm{d}$ & $\mathrm{C}$ & 3.830 & 0.998298 & 0.996177 & -0.213 & $\%$ \\
\hline C80100 & $\mathrm{pu}-\mathrm{d}$ & ti & 8.000 & 0.986907 & 0.984861 & -0.208 & 응 \\
\hline C 90100 & $\mathrm{pu}-\mathrm{d}$ & $\mathrm{W}$ & 4.700 & 0.992985 & 0.991153 & -0.185 & $\%$ \\
\hline c10010 & $\mathrm{pu}-\mathrm{d}$ & $u-235$ & 0.660 & 0.999219 & 0.996183 & -0.305 & $\%$ \\
\hline c11010 & $\mathrm{pu}-\mathrm{d}$ & $u-238$ & 1.930 & 0.992996 & 0.990539 & -0.248 & $\%$ \\
\hline C12010 & $\mathrm{pu}-\mathrm{d}$ & $u-238$ & 6.740 & 0.997434 & 0.994696 & -0.275 & $\%$ \\
\hline C13010 & $\mathrm{pu}-\mathrm{d}$ & $\mathrm{u}$ & 4.130 & 1.000410 & 0.997680 & -0.274 & $\%$ \\
\hline c14010 & $\mathrm{pu}-\mathrm{d}$ & $\mathrm{u}$ & 19.600 & 0.998271 & 0.994706 & -0.358 & $\%$ \\
\hline c10100 & $u-233$ & & & 0.996675 & 0.993851 & -0.284 & 응 \\
\hline c20100 & $u-233$ & be & 2.050 & 1.000000 & 0.996944 & -0.307 & $\%$ \\
\hline c30100 & $u-233$ & be & 4.200 & 1.002300 & 0.999646 & -0.265 & $\%$ \\
\hline c40100 & $u-233$ & $\mathrm{~W}$ & 2.440 & 0.998245 & 0.995478 & -0.278 & \% \\
\hline c50100 & $u-233$ & $\mathrm{~W}$ & 5.790 & 0.997022 & 0.994353 & -0.268 & $\%$ \\
\hline c 60100 & $u-233$ & $u-235$ & 1.210 & 1.001660 & 0.998594 & -0.307 & $\%$ \\
\hline c70100 & $u-233$ & $u-235$ & 1.980 & 1.005640 & 1.001760 & -0.387 & 응 \\
\hline C80100 & $u-233$ & $u-235$ & 4.820 & 1.009170 & 1.003760 & -0.539 & $\%$ \\
\hline C 90100 & $u-233$ & $\mathrm{u}$ & 2.300 & 1.002160 & 0.998909 & -0.325 & $\%$ \\
\hline c10010 & $u-233$ & $\mathrm{u}$ & 5.310 & 1.004550 & 1.001070 & -0.348 & $\%$ \\
\hline c11010 & $u-233$ & $\mathrm{u}$ & 19.910 & 1.002520 & 0.998268 & -0.426 & $\%$ \\
\hline $\mathrm{C} 001$ & $u-235$ & be & 1.27 & 0.991333 & 0.984829 & -0.660 & $\div$ \\
\hline $\mathrm{C} 002$ & $u-235$ & be & 2.54 & 0.993284 & 0.986833 & -0.654 & $\%$ \\
\hline $\mathrm{C} 003$ & $u-235$ & C & 1.27 & 1.002000 & 0.995486 & -0.654 & $\%$ \\
\hline $\mathrm{C} 004$ & $u-235$ & C & 2.54 & 1.003290 & 0.996490 & -0.682 & $\%$ \\
\hline $\mathrm{c} 005$ & $u-235$ & $\mathrm{mg}$ & 1.27 & 0.991813 & 0.985385 & -0.652 & $\%$ \\
\hline $\mathrm{C} 006$ & $u-235$ & $\mathrm{mg}$ & 2.54 & 0.994947 & 0.988433 & -0.659 & $\%$ \\
\hline $\mathrm{C} 007$ & $u-235$ & al & 1.27 & 0.989334 & 0.982866 & -0.658 & $\%$ \\
\hline $\mathrm{C} 008$ & $u-235$ & al & 2.54 & 0.988649 & 0.982307 & -0.646 & $\%$ \\
\hline c009 & $u-235$ & ti & 1.27 & 0.994135 & 0.987767 & -0.645 & $\div$ \\
\hline $\mathrm{C} 010$ & $u-235$ & ti & 2.54 & 0.996451 & 0.989906 & -0.661 & $\%$ \\
\hline $\mathrm{C} 011$ & $u-235$ & $\mathrm{fe}$ & 1.27 & 0.998081 & 0.991672 & -0.646 & $\%$ \\
\hline $\mathrm{C} 012$ & $u-235$ & $\mathrm{fe}$ & 2.54 & 0.990955 & 0.984332 & -0.673 & $\div$ \\
\hline $\mathrm{C} 013$ & $u-235$ & $\mathrm{ni}$ & 1.27 & 0.990904 & 0.984588 & -0.641 & $\%$ \\
\hline $\mathrm{C} 014$ & $u-235$ & $\mathrm{ni}$ & 2.54 & 0.994334 & 0.987967 & -0.644 & $\%$ \\
\hline C015 & $u-235$ & $\mathrm{cu}$ & 1.27 & 0.991635 & 0.985316 & -0.641 & $\%$ \\
\hline C016 & $u-235$ & $\mathrm{cu}$ & 2.54 & 0.998562 & 0.992127 & -0.649 & 응 \\
\hline $\mathrm{C} 017$ & $u-235$ & mo & 1.27 & 1.003360 & 0.996582 & -0.680 & $\%$ \\
\hline $\mathrm{C} 018$ & $u-235$ & mo & 2.54 & 1.013120 & 1.006210 & -0.687 & $\%$ \\
\hline C019 & $u-235$ & mo-al & & 1.003810 & 0.997282 & -0.655 & $\%$ \\
\hline $\mathrm{C} 020$ & $u-235$ & $\mathrm{~W}$ & 1.27 & 0.987416 & 0.980685 & -0.686 & $\%$ \\
\hline $\mathrm{C} 021$ & $u-235$ & $\mathrm{~W}$ & 2.54 & 0.987675 & 0.981390 & -0.640 & 응 \\
\hline c10100 & $u-235$ & & & 0.998285 & 0.992167 & -0.617 & $\%$ \\
\hline c20100 & $u-235$ & & & 1.004730 & 0.997933 & -0.681 & $\%$ \\
\hline c30100 & $u-235$ & & & 1.001100 & 0.994867 & -0.627 & 응 \\
\hline
\end{tabular}




$\begin{array}{lllllll}\mathrm{c} 40100 & \mathrm{u}-235 & \mathrm{be} & 2.222 & 0.998019 & 0.991442 & -0.663 \% \\ \mathrm{c} 5100 & \mathrm{u}-235 & \mathrm{be} & 3.260 & 0.999108 & 0.992707 & -0.645 \% \\ \mathrm{c} 60100 & \mathrm{u}-235 & \mathrm{be} & 4.710 & 1.002990 & 0.996677 & -0.633 \% \\ \mathrm{c} 70100 & \mathrm{u}-235 & \mathrm{be} & 5.440 & 1.000370 & 0.993858 & -0.655 \% \\ \mathrm{c} 80100 & \mathrm{u}-235 & \mathrm{be} & 9.270 & 1.001940 & 0.995947 & -0.602 \% \\ \mathrm{c} 90100 & \mathrm{u}-235 & \mathrm{be} & 11.790 & 1.001540 & 0.995308 & -0.626 \% \\ \mathrm{c} 10010 & \mathrm{u}-235 & \mathrm{c} & 10.160 & 0.997750 & 0.991028 & -0.678 \% \\ \mathrm{c} 11010 & \mathrm{u}-235 & \mathrm{c} & 15.240 & 0.993739 & 0.987280 & -0.654 \% \\ \mathrm{c} 12010 & \mathrm{u}-235 & \mathrm{ni} & 4.940 & 0.997086 & 0.990638 & -0.651 \% \\ \mathrm{c} 13010 & \mathrm{u}-235 & \mathrm{cu} & 5.030 & 1.003730 & 0.997110 & -0.664 \% \\ \mathrm{c} 14010 & \mathrm{u}-235 & \mathrm{cu} & 10.560 & 1.009160 & 1.002330 & -0.681 \% \\ \mathrm{c} 15010 & \mathrm{u}-235 & \mathrm{w} & 5.080 & 1.000260 & 0.993600 & -0.670 \% \\ \mathrm{c} 16010 & \mathrm{u}-235 & \mathrm{w} & 10.160 & 1.001170 & 0.994547 & -0.666 \% \\ \mathrm{c} 17010 & \mathrm{u}-235 & \mathrm{pb} & 8.990 & 1.020890 & 1.014200 & -0.660 \% \\ \mathrm{c} 18010 & \mathrm{u}-235 & \mathrm{pb} & 17.220 & 1.020590 & 1.013680 & -0.682 \% \\ \mathrm{c} 19010 & \mathrm{u}-235 & \mathrm{u} & 1.760 & 1.001340 & 0.994603 & -0.677 \% \\ \mathrm{c} 20010 & \mathrm{u}-235 & \mathrm{u} & 4.470 & 1.005410 & 0.998442 & -0.698 \% \\ \mathrm{c} 21010 & \mathrm{u}-235 & \mathrm{u} & 9.960 & 1.002660 & 0.995373 & -0.732 \% \\ \mathrm{c} 22010 & \mathrm{u}-235 & \mathrm{u} & 18.010 & 0.998976 & 0.991576 & -0.746 \% \\ & & & \text { Averages } & 0.999340 & \mathbf{0} .994163 & -0.521 \%\end{array}$




\section{Appendix D: Summary of New Conventions and Options}

These are NEW for TART2005; there are no changes for TART2012

To help explain and illustrate the use of the new options the TART2005 DVD distribution includes example input decks. I encourage you to use TARTCHEK to look at these examples - particularly using 3-D views, so you can see them better.

See chapter 2, on Input Parameters for details. Here I only briefly list the new input options and conventions.

Cubic surface

xcubic nb x0 y0 z0 d c b a ycubic nb x0 y0 z0 d c b a zcubic nb x0 y0 z0 d c b a

Torus

xtorus nb x0 y0 z0 a b c ytorus nb x0 y0 z0 a b c ztorus nb x0 y0 z0 a b c

Macro surfaces: boxes, cylinders and cones with finite limits

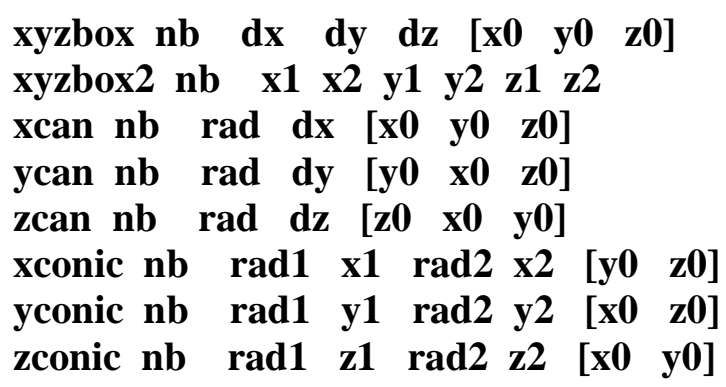

Macro volumes: simplified input for complicated surfaces

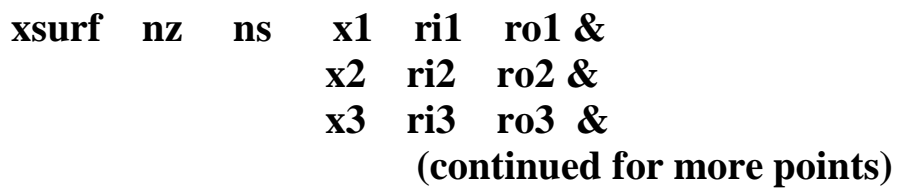




$\begin{array}{llllll}\text { zsurf } & \text { nz } & \text { ns } & \text { z1 } & \text { ri1 } & \text { ro1 \& } \\ & & & \text { z2 } & \text { ri2 } & \text { ro2 \& } \\ & & \text { z3 } & \text { ri3 } & \text { ro3 \& } \\ & & & \text { (continued for more points) }\end{array}$

Rotation about the $\mathrm{X}, \mathrm{Y}$ or $\mathrm{Z}$ axis

xrotate ang is1 thru is 2

xrotate ang is1 is 2 is $3 . . . .$.

yrotate ang is 1 thru is 2

yrotate ang is1 is 2 is $3 . . . . .$.

zrotate ang is1 thru is2

zrotate ang is1 is 2 is 3 ......

Translation of Spatial Coordinates

addxyz xadd yadd zadd is1 thru is 2

addxyz xadd yadd zadd is 1 is 2 is 3 ................

Cloning (Duplicating) Surfaces

clones ns is1 thru is2

clones ns is 1 is 2 is 3 .....

Reduced, Reflecting Geometry

xabove $x 0$

yabove y0

zabove $\mathrm{z0}$

xbelow $\mathbf{x 0}$

ybelow y0

zbelow z0

New Sources

source19 (spherical shell), source 20 (cylindrical shell), and source21 (a rectangular box) allow you to sample from irregularly shaped zones,

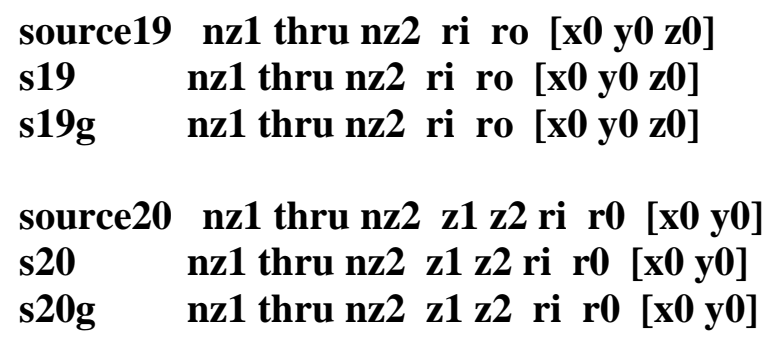




\section{source21 nz1 thru nz2 x1 x2 y1 y2 z1 z2 \\ s21 nz1 thru nz2 x1 x2 y1 y 2 z1 z2 \\ s21g nz1 thru nz2 x1 x2 y1 y2 z1 z2}

Defining Zone Volume by User Input

volume nz1 vz1 nz2 vz2 nz3 vz3.....

\section{Changes in sentinels}

“BEST" Options, now set for you

Sentinels 20 (self-shielding), 25 (fluorescence) and 39 (thermal scattering) are now all initialized as ON. You should NEVER use/change these unless you really do want to turn off this physics. Starting with TART2005, if a continuous energy cross section file (named NEUTRON) is present Sentinel 20 defaults to 2, to use this data; for compatibility with older TART data libraries that do not include continuous energy cross sections, if file NEUTRON is not present sentinel 20 defaults to 1, to use multi-band parameters.

Sentinels 8, 9, 13,14, 15 and 16, limit the energy range for tracking and tallying neutrons and photons. These are now automatically set for you, and you should NEVER use these unless you really do want to limit these ranges.

\section{New Sentinels, 54 through 57}

54 - definition of the number of neutrons per fission; use either $<v>$, or sample the probability distribution of $v$.

55 - define a correlated spontaneous fission source.

56 - what to tally with tally types 11 and 12: neutrons entering a zone, absorption, capture, fission....

57 - use either total or prompt $<v>$. Starting with TART2005, this option is also used to control the energy and time dependence of delayed neutrons; it now defaults to 2 , to use the delayed neutron energy spectra, but all neutrons are produced promptly (immediately); for time dependence use 3 for this option. 


\section{References}

[1r] TART2005: A Coupled Neutron-Photon 3-D, Time Dependent, Combinatorial Geometry Monte Carlo Transport Code," Lawrence Livermore National Laboratory, UCRL-SM-218009, November 22, 2005

[1] "TART95: A Coupled Neutron-Photon Monte Carlo Transport Code," Lawrence Livermore National Laboratory, UCRL-MA-121319, July 1995, by D.E. Cullen, A.L. Edwards and E.F. Plechaty

[2] "TART96: A Coupled Neutron-Photon 3-D, Combinatorial Geometry Monte Carlo Transport Code," Lawrence Livermore National Laboratory, UCRL-ID-126455, November, 1996, by D.E. Cullen.

[3] “ PREPRO 2010: 2010 ENDF/B Pre-Processing Codes”, IAEA-NDS-39, Rev. 14, October 2010, by D.E. Cullen.

[4] "THERMAL: A Routine Designed to Calculate Neutron Thermal Scattering," Lawrence Livermore National Laboratory, UCRL-ID-120560-Rev-1, Sept. 1995, by D.E. Cullen.

[5] "POINT 2012: ENDF/B-VII.1 Final Temperature Dependent Cross Section Library", LLNL-TR-534938, January 2012, by D.E. Cullen

[6] "EPDL97: the Evaluated Photon Data Library, '97 Version," Lawrence Livermore National Laboratory, UCRL--50400, Vol. 6, Rev. 5, by D.E. Cullen; this is now the official ENDF/B-VI photon interaction data library.

[7] "Nuclear Data Preparation", pp. 279-425, Vol. 1, in "The Handbook of Nuclear Engineering", Springer Publishing, NY, NY (2010), by D.E. Cullen

[8a] "TART97: A Coupled Neutron-Photon 3-D, Combinatorial Geometry Monte Carlo Transport Code," Lawrence Livermore National Laboratory, UCRL-ID-126455, Rev. 1, November, 1997, by D.E. Cullen

[8b] "TART98: A Coupled Neutron-Photon 3-D, Combinatorial Geometry Time Dependent Monte Carlo Transport Code," Lawrence Livermore National Laboratory, UCRL-ID-126455, Rev. 2, November, 1998, by D.E. Cullen

[8c] "TART2000: A Coupled Neutron-Photon 3-D, Combinatorial Geometry Time Dependent Monte Carlo Transport Code," Lawrence Livermore National Laboratory, UCRL-ID-126455, Rev. 3, November, 2000, by D.E. Cullen

[8d] "TART2002: A Coupled Neutron-Photon 3-D, Combinatorial Geometry Time Dependent Monte Carlo Transport Code," Lawrence Livermore National Laboratory, UCRL-ID-126455, Rev. 4, June, 2003, by D.E. Cullen 
[9] "A Simple Model of Photon Transport", by D.E. Cullen, Nuclear Instrumentation and Methods in Physics Research B101 (1995) pp 499-510. The original extended form of this paper is now available on-line at my personal website,

http://home.comcast.net/ redcullen1

[10] Private Communication, (1988), by R.J. Howerton

[11] "New Thermal Neutron Scattering Files for ENDF/B-VI Release 2", Los Alamos National Laboratory (March 1994), by R.E. MacFarlane

[12] To contact the author,

Dermott E. Cullen

1466 Hudson Way

Livermore, CA 94550

Tele: 925-443-1911

E.Mail: RedCullen1@comcast.net

Website: http://home.comcast.net/ redcullen1 
Lawrence Livermore National Laboratory

Technical Information Department

Livermore, CA 94551 